\title{
JNS Review Recognition 2020
}

Journal of Nutritional Science (2020), vol. 9, e54, page 1 of 1

doi:10.1017/jns.2020.48

The Editorial Board of the Journal of Nutritional Science would like to thank the following for their contribution as peer reviewers in 2020:

Edyta Adamska

Folasade Adebayo

Demetrius Albanes

Meshari Alkoot

Naser Alsharairi

Giannis Arnaoutis

Zahra Bahadoran

Emma Baldry

Guillermina Barril-Cuadrado

Eirini Bathrellou

Carolina Berchieri- Ronchi

Anne Bjerregaard

Petra Brembeck

Magritt Brustad

Jennifer Carter

Muhammad Chacher

Lieselotte Cloetens

Peter Curtis

Sara da Silva

Luana Azevedo de Aquino

Bethelhem Debela

Hans Demmelmair

Arvind Diwan

Saeid Doaei

Gudina Egata

Arja Erkkilä

Edna Gamboa

Michael Georgoulis

Admasu Gizaw

Beatriz Gonçalves

Bartira Gorgulho
Horst Göring

Jessica Gwin

Stephen Hennigar

Javad Heshmati

Andrew Hill

Iva Hojsak

Paul Hulshof

Aiko Hyakutake

Francois Iris

Patricia Jaime

Niina Kaartinen

Russell Kabir

Satish Kalhan

Nitin Kapoor

J. Philip Karl

Maryam Khan

James King

Mirit Kisner

David Kitts

Yoko Komada

Patrícia Lisboa

Tahereh Mahammadabadi

Simonette Mallard

Eirini Mamalaki

Y. Fabiola Marquez-Sandoval

Koutatsu Maruyama

Velimir Matkovic

Walter Mihatsch

Arlene Mitchell

Maedeh Moradi

Karen Murphy
Tzortzis Nomikos

Meghan O’Neill

Archana Panche

Jaqueline Pereira

Marcos Pereira-Santos

Elsa Pinto

Alex Rafacho

Alanderson Ramalho

Stina Ramne

Elisabet Rothenberg

Lisa Ryan

Aki Saito

Jonathan Scott

Harbindar Singh

Chelsea Singleton

Regina Spadari-Bratfisch

Zhihong Sun

Giovanni Tarantino

Lílian Gonçalves Teixeira

Paul Trayhurn

Konstantinos Tziomalos

Pedro Urriola

Simone Vargas

Clemens von Schacky

Yeli Wang

Leigh Ward

Kurt Widhalm

Lynda Williams

Katsuhiko Yokoi

Katherine Younger

Jing Yuan

(C) The Author(s), 2020. Published by Cambridge University Press on behalf of The Nutrition Society. This is an Open Access article, distributed under the terms of the Creative Commons Attribution licence (http://creativecommons.org/licenses/by/4.0/), which permits unrestricted re-use, distribution, and reproduction in any medium, provided the original work is properly cited. 